\title{
Tomita's Spectral Analysis in Krein Spaces
}

\author{
By \\ Yoshiomi NAKAGAMI*
}

\section{§1. Introduction}

The most manageable indefinite inner product space is a Pontrjagin space or a more general Krein space. The spectral analysis of operators on indefinite inner product spaces is known to be difficult even in such spaces. Tomita has proposed one formulation of the spectral decomposition for bounded selfadjoint operators with respect to the indefinite inner product as follows: Every bounded selfadjoint operator on a Pontrjagin space with respect to the indefinite inner product is expressed in the form

$$
h=\left(\begin{array}{ccc}
h_{11} & h_{12} & h_{13} \\
0 & h_{22} & h_{23} \\
0 & 0 & h_{33}
\end{array}\right)
$$

where $h_{11}$ and $h_{33}$ act on neutral subspaces and $h_{22}$ is bounded and selfadjoint with respect to some positive definite inner product of the Hilbertian structure. For many applications it is desirable to extend the operator and the space to an unbounded operator as well as to a Krein space. A unitary operator with respect to the indefinite inner product is bounded on a Pontrjagin space, but not on a Krein space. Therefore it gives rise to another problem which must be solved concerning the matrix representation and the product of unbounded operators as in (1.1) and (1.2) below. To avoid such troubles we will modify our problem and consider a bounded unitary operator instead of a selfadjoint operator with respect to the indefinite inner product. As a result we can show

Communicated by H. Araki, July 24, 1985. Revised January 27, 1986.

* Department of Mathematics, Yokohama City University, 22-2 Seto, Kanazawa-ku, Yokohama, 236 Japan. 
Theorem. If $u$ is a bounded unitary on a Krein space $\Re$ with $u$-JuJ compact, then there exists a unitary (not necessarily bounded) operator $v$ with respect to the indefinite inner product and a dense subspace $D$ of $\Re$ such that

$$
v u v^{-1}=\left(\begin{array}{ccc}
u_{11} & u_{12} & u_{13} \\
0 & u_{22} & u_{23} \\
0 & 0 & u_{33}
\end{array}\right) \text { on } D,
$$

where $u_{11}$ and $u_{33}$ act on neutral subspaces and $u_{22}$ is a unitary with respect to a positive definite inner product of the Hilbertian structure.

A slightly more precise statement will be given in Theorem 3.1. In $\S 2$ we shall prepare definitions on indefinite inner product spaces and recall some related results. Especially the concept for a \#-unitary to be quasi-\#-spectral is important. Our main assertion is Theorem 3.1. In $\S 4$ we will give supplementary discussions for the theorem. First we will give an example of a quasi-\#-spectral bounded \#-unitary whose spectrum is distributed to the outside of the unit circle. This shows that the spectrum is not preserved by the quasi-\#-spectrality unlike the \#-spectrality. Secondly, in order to see the difference of our result from that of Krein-Langer explained in [6] we will restrict ourselves to a finite dimensional space and give a concrete form of a Tomita's triangular matrix for a \#-unitary. Finally, we will give a sufficient condition that a Tomita's triangular matrix is decomposed into the direct sum of the $(1,1)+(3,3)$ component and the $(2,2)$ component.

This paper is a detailed proof of a part of our results in [8]. The same type of analysis for \#-selfadjoint operators in Krein spaces will appear in a separate paper, in which we will use our theorem.

\section{§2. Preliminary}

In this paper we shall use the traditional terminologies and notations in the theory of Hilbert spaces.

Throughout this paper $\AA$ will denote a complex Hilbert space with respect to a positive definite inner product $(\mid)$. 
Definition 2.1. A pair $\{\Re,\langle\rangle$,$\} of a Hilbert space \Omega$ and a continuous indefinite inner product $\langle$,$\rangle is called a Krein space if$ the operator $J$ defined by $\langle\xi, \eta\rangle=(J \xi \mid \eta)$ for all $\xi$, $\eta$ in $\Omega$ is (selfadjoint and) unitary.

We sometimes use the notation $\{\Omega, J\}$ for Krein spaces. We denote the spectral decomposition of $J$ by $J^{+}-J^{-}\left(J^{+}+J^{-}=1\right)$ and the corresponding subspaces by $\Re^{ \pm}=J^{ \pm}$. The operator $J$ is called a metric operator.

Definition 2.2. A Krein space $\{\Re,\langle\rangle$,$\} is called a Pontrjagin$ space if $\operatorname{dim} \Re^{+}$or $\operatorname{dim} \Re^{-}$is finite.

A vector $\xi$ in $\Omega$ is called pasitive, neutral or negative according as $\langle\xi, \xi\rangle$ is positive, zero or negative. The same terminologies will be used for subspaces and for projections.

The adjoint $x^{\#}$ of a densely defined linear operator $x$ with respect to the indefinite inner product is defined similarly as the case of a positive definite inner product:

$$
\langle x \xi, \eta\rangle=\left\langle\xi, x^{\sharp} \eta\right\rangle \text { for } \xi \in D(x) \text { and } \eta \in D\left(x^{\sharp}\right) \text {. }
$$

The domain $D\left(x^{\sharp}\right)$ of $x^{\sharp}$ is the set of all $\eta$ such that the mapping $\xi \in D(x) \mapsto\langle x \xi, \eta\rangle \in \mathbb{C}$ is continuous. Therefore $x^{\sharp}=J x^{*} J$ and $D\left(x^{\sharp}\right)$ $=J D\left(x^{*}\right)$. The \#-adjoint operation satisfies the condition of the involution for bounded operators:

$$
(x+y)^{\sharp}=x^{\sharp}+y^{\sharp},(\lambda x)^{\sharp}=\bar{\lambda} x^{\sharp},(x y)^{\sharp}=y^{\sharp} x^{\sharp} 。
$$

A densely defined operator $x$ satisfying $x=x^{\#}$ is said to be \#-selfadjoint. A \#-selfadjoint operator defined everywhere is called a \#-projection if it is idempotent. Since it is closed, it is bounded and the range is closed. A densely defined operator $v$ satisfying $\langle v \xi$, $v \eta\rangle=\langle\xi, \eta\rangle$ for all $\xi, \eta$ in $D(v)$ is called a \#-isometry. Since $D\left(v^{\sharp} v\right)=D(v)$ as easily seen from the definition, $v$ is \#-isometry if and only if $v^{\sharp} v=1$ on $D(v)$. A closed \#-isometry $v$ is called a \#-unitary if the range $R(v)$ is dense in $\Omega$ and $v^{\#}=v^{-1}$. If $v$ is $\#$-unitary, then $v^{\#}$ is also \#-unitary. A \#-unitary is bounded if and only if $v^{\sharp} v=v v^{\sharp}=1$. On the Pontrjagin space a \#-unitary is automatically bounded, [4]. On the contrary there exists an unbounded \#-unitary on a Krein space. For example, let $p$ be a neutral projection such that $p=\sum_{j=1}^{\infty} p_{j}$ and $\operatorname{dim} p_{j}=1$ for 
all $j \in N$. Then

$$
u=\sum_{j=1}^{\infty}\left(j p_{j}+j^{-1} p_{j}^{\#}\right)+\left(1-p-p^{\#}\right)
$$

is an unbounded \#-unitary. Other examples are given in [3]. Here we must apologize for our notations and terminologies. The traditional terminologies for "selfadjoint", "unitary", etc., with respect to the indefinite inner product space are " $J$-selfadjoint", " $J$-unitary", etc. When we fix an indefinite inner product on a complex vector space, a metric operator $J$ depends on the choice of a positive definite inner product which makes $\Omega$ a Krein space, while the adjoint with respect to the indefinite inner product is decided independently from it. This fact makes us employ the symbol \# instead of $J$, following Tomita as in [11].

A bounded \#-selfadjoint (resp. \#-unitary) operator $x$ on a Pontrjagin space is called \#-spectral if there exists a \#-unitary $v$ such that $v x v^{\#}$ is selfadjoint (resp. unitary), [11]. We shall extend this concept to a Krein space.

Definition 2.3. A bounded \#-unitary $u$ on a Krein space is called $\#$-spectral if there exists a bounded \#-unitary $v$ such that $v u v^{\#}$ is unitary.

To give a weaker but more important concept we shall introduce a set

$$
D_{0}=v D\left(v^{*} v\right)=v^{*-1} R\left(v^{*} v\right)
$$

for a \#-unitary $v$. Then $D_{0}$ is dense in $\AA$. Indeed, since $D\left(v^{*} v\right)$ is a core of $v, v D\left(v^{*} v\right)$ is dense in $R(v)$, and hence in $\Omega$.

Definition 2.4. A \#-unitary $u$ on a Krein space is called quasi\#-spectral if there exists a \#-unitary $v$ such that

(i) $D\left(v^{*} v\right) \subset D(u)$ and $u D\left(v^{*} v\right)=D\left(v^{*} v\right)$,

(ii) $v u v^{\sharp} \mid D_{0}$ is closable and the closure is unitary.

In the above definition, $J u^{-1} J=u^{*}$ and $J|v| J=|v|^{-1}$. Hence $J R(u)$ $=D\left(u^{*}\right)$ and $J R\left(v^{*} v\right)=D\left(v^{*} v\right)$. Condition (i) implies

and

$$
R\left(v^{*} v\right)=J D\left(v^{*} v\right) \subset J R(u)=D\left(u^{*}\right)
$$

$$
u^{*} R\left(v^{*} v\right)=J u^{-1} J R\left(v^{*} v\right)=J u^{-1} D\left(v^{*} v\right)=J D\left(v^{*} v\right)=R\left(v^{*} v\right) .
$$


Since $\left\langle v u v^{\sharp} \xi, v u v^{\sharp} \eta\right\rangle=\langle\xi, \eta\rangle$ for $\xi, \eta \in D_{0}$ and the closure $w$ of $v u v^{\sharp} \mid D_{0}$ is bounded, $w$ is \#-unitary as well as unitary. Bofore going into our main discussion we shall recall the Tomita's theorem, which motivates this paper.

Theorem 2.5 ([10]). Let $\{\AA,\langle\rangle$,$\} be a Pontrjagin space. If h$ is a bounded \#-selfadjoint operator, then there exists a neutral projection $p$ such that $p \Re$ is invariant under $h$ and $\left(1-p-p^{\#}\right) h\left(1-p-p^{\#}\right)$ is \#spectral.

A projection $p$ is neutral if and only if $p p^{\sharp}\left(=p^{\sharp} p\right)=0$. Therefore if bounded operators $x$ and $x^{\sharp}$ leave a neutral subspace $p \AA$ invariant, then $x$ is represented in the form of a triangular matrix

$$
\left(\begin{array}{ccc}
x_{11} & x_{12} & x_{13} \\
0 & x_{22} & x_{23} \\
0 & 0 & x_{33}
\end{array}\right)
$$

where $p_{1}=p, p_{2}=1-p-p^{\sharp}, p_{3}=p^{\sharp}$ and $x_{i j}$ is the restriction of $p_{i} x$ to $p_{j} \Omega$. In this case, $J$ is also represented in the form

$$
\left(\begin{array}{ccc}
0 & 0 & J_{13} \\
0 & J_{22} & 0 \\
J_{31} & 0 & 0
\end{array}\right)
$$

and $J_{22}$ is a selfadjoint unitary on $p_{2} \Re$.

Definition 2.6. The above triangular matrix (2.2) is called a Tomita's triangular matrix for $x$ if $x_{22}$ is quasi-\#-spectral on $\left\{p_{2} \Re, J_{22}\right\}$.

The above Tomita's theorem is then restated in such a way that any bounded \#-selfadjoint operator on a Pontrjagin space is represented by a Tomita's triangular matrix and the $(2,2)$ component is \#spectral.

For the proof of the theorem we approximate the given bounded \#-selfadjoint operator by a net of \#-selfadjoint operators on finite dimensional Krein spaces, on which we prove the theorem. Then we replace the \#-unitary implementing the \#-spectrality by an operator $T$ in the unit ball as in Lemma 2.7 in below and then manipulate the limit procedure. In this sense the discovery of the operator $T$ is crucial in Tomita's spectral analysis. 
Lemma 2.7 ([11]). For a bounded \#-selfadjoint operator $h$ on a Pontrjagin space the following two conditions are equivalent:

(i) $h$ is \#-spectral; and

(ii) there exists an operator $T$ such that

a) $0<T<1$

b) $J T J=1-T$

c) $T h(1-T)$ is selfadjoint.

\section{§3. Tomita's Triangular Matrix for \#-Unitaries}

In this section we shall generalize the Tomita's spectral analysis for bounded \#-selfadjoint operators in a Pontrjagin space to that for bounded \#-unitaries in a Krein space, and show a similar assertion to Theorem 2.1 for them.

Theorem 3.1. If $u$ is a bounded \#-unitary in a Krein space with $J^{+} u J^{-}$compact, then $u$ is represented by a Tomita's triangular matrix. If $\Re$ is a Pontrjagin space, then $u_{22}$ is \#-spectral.

The compactness of $J^{+} u J^{-}$is equivalent to that of $u-J u J$. This implies that $u^{*} u-1=\left(u^{*}-u^{*}\right) u$ and $u u^{*}-1=u\left(u^{*}-u^{\#}\right)$ are compact.

For the proof of this theorem we must prepare the following five lemmas.

Lemma 3. 2. A bounded \#-unitary $u$ of the form $\mu 1+k$ with $\mu \in \mathbb{C}$, $|\mu|=\mathrm{I}$ and $k \in \mathscr{L}(\Re)$ of finite rank is represented by a Tomita's triangular matrix.

Proof. Among neutral projections invariant under $u$ and $u^{\sharp}$, let $p$ be a maximal one. Then $u^{\prime}=\left(1-p-p^{\#}\right) u \mid\left(1-p-p^{\#)} \Omega\right.$ is a \#-unitary on the subspace $\left(1-p-p^{\sharp}\right) \Re$ and has no nonzero neutral projections

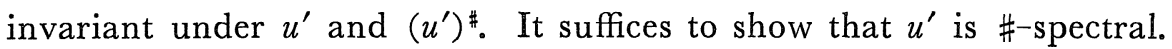
Therefore we may assume that $u$ itself has no nonzero neutral projections invariant under $u$ and $u^{\sharp}$.

To decompose $u$ into the generalized eigenspaces, let $\operatorname{Sp}(u)$ be the spectrum of $u$. Since $k$ is of finite $\operatorname{rank}, \operatorname{Sp}(u)$ consists of a finite number of points. Put 


$$
e(\lambda)=\frac{1}{2 \pi i} \int_{C_{\lambda}}(z 1-u)^{-1} d z \quad \text { for } \quad \lambda \in \operatorname{Sp}(u)
$$

where $C_{\lambda}$ is a small circle with center $\lambda$ and oriented counter clockwise. Then each $e(\lambda)$ satisfies that
a) $e(\lambda)$ commutes with $u$
b) $e(\lambda) e\left(\lambda^{\prime}\right)=\delta_{\lambda, \lambda^{\prime}} e(\lambda)$
c) $\sum_{\lambda \in \mathrm{Sp}(u)} e(\lambda)=1$
d) $(u-\lambda 1) e(\lambda)$ is nilpotent.

Furthermore, $e(\lambda)$ satisfies

$$
\text { e) } e(\lambda)^{\#}=e\left(\bar{\lambda}^{-1}\right) \text {. }
$$

Indeed, for any $\xi, \eta$ in $\Omega$, we have

$$
\left\langle e(\lambda)^{\# \xi}, \eta\right\rangle=\overline{\langle e(\lambda) \eta, \xi\rangle}=\left(\frac{1}{2 \pi i} \int_{c_{\lambda}}\left\langle(z 1-u)^{-1} \eta, \xi\right\rangle d z\right)^{-} 。
$$

We use the fact that

$$
\left(\int_{C_{\lambda}} f(z) d z\right)^{-}=-\int_{C_{\bar{\lambda}}} \overline{f(\bar{z})} d z
$$

The right hand side of (3.2) turns out to be

$$
\frac{1}{2 \pi i} \int_{C_{\bar{\Sigma}}} \overline{\left\langle(\bar{z} l-u)^{-1} \eta, \xi\right\rangle} d z=\frac{1}{2 \pi i} \int_{C_{\bar{\Sigma}}}\left\langle\xi,(\bar{z} l-u)^{-1} \eta\right\rangle d z \text { 。 }
$$

Since $\left(x^{\sharp}\right)^{-1}=\left(x^{-1}\right)^{\sharp}$ for any invertible $x$, this is equal to

$$
\begin{aligned}
& \left.\frac{1}{2 \pi i} \int_{C_{\bar{\Sigma}}}\left\langle z 1-u^{-1}\right)^{-1} \xi, \eta\right\rangle d z \\
& \quad=\frac{1}{2 \pi i} \int_{C_{\bar{\Sigma}}-1}\left\langle-\left(z^{-1} 1-u^{-1}\right)^{-1} \xi, \eta\right\rangle z^{-2} d z \\
& \quad=\frac{1}{2 \pi i} \int_{C_{\bar{\lambda}}-1}\left\langle z^{-1} u(z 1-u)^{-1} \xi, \eta\right\rangle d z \\
& \quad=\frac{1}{2 \pi i} \int_{C_{\bar{\lambda}}-1}\left\langle z^{-1}(z 1-u)^{-1} \xi, u^{\sharp} \eta\right\rangle d z \\
& =\left\langle u^{-1} e\left(\bar{\lambda}^{-1}\right) \xi, u^{-1} \eta\right\rangle=\left\langle e\left(\bar{\lambda}^{-1}\right) \xi, \eta\right\rangle .
\end{aligned}
$$

Therefore $\lambda \in \operatorname{Sp}(u)$ implies $\bar{\lambda}^{-1} \in \operatorname{Sp}(u)$ by e). If $|\lambda| \neq 1$, then $\lambda \neq \bar{\lambda}^{-1}$ and hence $e(\lambda)^{\#} e(\lambda)=0$. This means that $e(\lambda) \Re$ is neutral. This case dose not occur by our assumption. Thus $|\lambda|=1$ and hence $e(\lambda)$ is a \#-projection for each $\lambda$ in $\operatorname{Sp}(u)$.

Next we shall show that $e(\lambda) \Omega$ is either positive or negative. For this it suffices to show that the generalized eigenspace $e(\lambda) \Omega$ for 
$u$ turns out to be the eigenspace. Because every neutral subspace of $e(\lambda) \Re$ is then invariant under $u$ and $u^{\#}\left(=u^{-1}\right)$, and hence it must be $\{0\}$ by assumption. Since $(u-\lambda 1) e(\lambda)$ is nilpotent, we can find the least positive integer $n$ such that

$$
(u-\lambda 1)^{n-1} e(\lambda) \neq 0 \text { and }(u-\lambda 1)^{n} e(\lambda)=0 .
$$

If $n>1$, there exists a nonzero $\eta$ of the form $(u-\lambda 1)^{n-1} \xi$ with $e(\lambda) \xi=\xi$. Then $u \eta=\lambda \eta$ and so $u^{\sharp} \eta=\bar{\lambda} \eta$. Therefore

$$
\langle\eta, \eta\rangle=\left\langle\eta,(u-\lambda 1)^{n-1} \xi\right\rangle=\left\langle\left(u^{\sharp}-\bar{\lambda} 1\right) \eta,(u-\lambda 1)^{n-2} \xi\right\rangle=0 .
$$

Thus $C \eta$ is a nonzero neutral subspace of $e(\lambda) \Re$ invariant under $u$ and $u^{\sharp}$. This contradicts our assumption.

Let $e^{+}\left(\right.$resp. $\left.e^{-}\right)$be the sum of \#-projections $e(\lambda)$ such that $e(\lambda) \Re$ is positive (resp. negative). Then $e^{ \pm}$are \#-projections with $e^{+}+e^{-}=1$. Thus there exists a bounded \#-nuitary $v$ such that $v e^{ \pm} v^{-1}=J^{ \pm}$. Then $v u v^{\#}$ commutes with $J$ and hence it is unitary. $\quad$ Q. E. D.

Lemma 3. 3. For a bounded \#-unitary $u$ the following two conditions are equivalent:

(i) $u$ is quasi-\#-spectral; and

(ii) there exists an operator $T \in \mathscr{L}(\Re)$ such that
a) $0<T<1$
b) $J T J=1-T$
c) $T u^{\sharp}(1-T)=(1-T) u^{*} T$.

Proof. (i) $\Rightarrow$ (ii). If $u$ is quasi-\#-spectral, there exists a \#-unitary $v$ such that

d) $u D\left(v^{*} v\right)=D\left(v^{*} v\right)$,

e) $v u v^{-1} \mid D_{0}$ is closable and the closure is unitary, where $D_{0}$ is defined by (2.1).

We denote $v u v^{-1} \mid D_{0}$ by $w$. Then $w$ is a bijection of $D_{0}$ onto itself by d). Since the closure $w^{-}$is unitary by e), $v u^{-1} v^{-1}=w^{*}$ on $D_{0}$ and hence $v u^{-1}=w^{*} v$ on $D\left(v^{*} v\right)$. Since

$$
v u^{-1} D\left(v^{*} v\right)=v D\left(v^{*} v\right) \subset D\left(v^{*}\right)
$$

by $d$ ), it follows that

$$
v^{*} v u^{\sharp}=v^{*} w^{*} v \quad \text { on } \quad D\left(v^{*} v\right) \text {. }
$$


If $\xi$ and $\eta$ are in $D\left(v^{*} v\right)$, then

$$
\begin{aligned}
\left(v^{*} w^{*} v \xi \mid \eta\right) & =\left(w^{*} v \xi \mid v \eta\right)=(v \xi \mid w v \eta)=(\nu \xi \mid v u \eta) \\
= & \left(v^{*} \xi \mid u \eta\right)=\left(u^{*} v_{v} \xi \mid \eta\right) .
\end{aligned}
$$

Therefore we have

$$
v^{*} v u^{\sharp}=u^{*} v^{*} v \quad \text { on } \quad D\left(v^{*} v\right) \text {. }
$$

Now we denote $v^{*} v\left(1+v^{*} v\right)^{-1}$ by $T$. Then $D(T)=\Re$ and $T$ satisfies $0 \leq T \leq 1$. Since

$$
J v^{*} v J=v^{\sharp}\left(v^{\sharp}\right)^{*}=v^{-1}\left(v^{*}\right)^{-1}=\left(v^{*} v\right)^{-1},
$$

$T$ satisfies b). Since Ker $v=\{0\}$, it follows that $T>0$. Combining this inequality with b), we see that $T$ satisfies a). Since (3.4) implies

$$
\left(1+v^{*} v\right)^{-1} v^{*} v u^{\sharp}\left(1+v^{*} v\right)^{-1}=\left(1+v^{*} v\right)^{-1} u^{*} v^{*} v\left(1+v^{*} v\right)^{-1}
$$

on $\Re=\left(1+v^{*} v\right) D\left(v^{*} v\right)$, c) follows.

(ii) $\Rightarrow$ (i). Using the operator $T$ satisfying conditions a), b) and c), we set

$$
v=\left\{T(1-T)^{-1}\right\}^{1 / 2}
$$

Condition b) implies

$$
J v^{2} J=J T(1-T)^{-1} J=(1-T) T^{-1}=v^{-2}
$$

and so $\left(v^{\sharp}\right)^{2}=v^{-2}$. Since $v$ is positive and selfadjoint, we obtain $v^{\#}$ $=v^{-1}$ and hence $v$ is $\#$-unitary.

Next we shall show d). Since the range of the left hand side of c) is contained in $R(1-T)$ and $1-T$ is invertible, we see that $(1-T)^{-1} T u^{\sharp}(1-T)=u^{*} T$, so that

$$
(1-T)^{-1} T u^{\sharp}=u^{*} T(1-T)^{-1} \quad \text { on } \quad R(1-T) \text {. }
$$

Since $R(1-T)=D\left(v^{2}\right)$, this turns out to be

$$
v^{2} u^{\sharp}=u^{*} v^{2} \quad \text { on } \quad D\left(v^{2}\right) \text { 。 }
$$

This shows that $u^{-1} D\left(v^{2}\right) \subset D\left(v^{2}\right)$. Since c) is equivalent to $T u(1-T)$ $=(1-T) u^{\sharp * T}$, it follows from the same discussion replaced $u$ by $u^{\sharp}$ that $v^{2} u=u^{\sharp *} v^{2}$ on $D\left(v^{2}\right)$. Thus $u D\left(v^{2}\right) \subset D\left(v^{2}\right)$. Consequently, we have $d$ ).

Finally we shall show e). We notice that $D_{0} \subset D(v) \cap R(v)$. The operator $w=v u v^{-1} \mid D_{0}$ satisfies $w^{-1}=v u^{\sharp} y^{-1} \mid D_{0}$. Equation (3.5) implies

$$
v u^{\#} v^{-1}=v^{-1} u^{*} v \text { on } D_{0} \text {. }
$$


If $\xi$ and $\eta$ are in $D_{0}$, then $u v^{-1} \eta \in D\left(v^{2}\right)(\subset D(v))$ and

$$
\left(v^{-1} u^{*} v \xi \mid \eta\right)=\left(u^{*} v \xi \mid v^{-1} \eta\right)=\left(v \xi \mid u v^{-1} \eta\right)=\left(\xi \mid v u v^{-1} \eta\right) .
$$

Therefore $w^{-1}=w^{*}$ on $D_{0}$ and $w$ is closable. Since $\left(w^{*} \xi \mid w^{*} \eta\right)=(\xi \mid \eta)$ for all $\xi, \eta$ in $D_{0}$ and $w^{*}$ is closed, we find that $D\left(w^{*}\right)=\Re$ and $w^{*}$ is unitary. Thus $w^{-}=w^{* *}=\left(w^{*}\right)^{-1}$ is unitary.

According to this lemma we can obtain the following lemma.

Lemma 3.4. For a bounded \#-unitary $u$ the following two conditions are equivalent:

(i) $u$ is represented by a Tomita's triangular matrix; and

(ii) there exists an operator $T \in \mathscr{L}(\Re)$ satisfying
a) $0 \leq T \leq 1$
b) $J T J=1-T$
c) $T u^{\sharp}(1-T)=(1-T) u^{*} T$.

Proof. (i) $\Rightarrow$ (ii). If we represent $u$ by a Tomita's triangular matrix, then $u_{22}$ is quasi-\#-spectral. By virtue of Lemma 3.3, there exists an operator $T_{22}$ on the Krein space $\left\{p_{2} \Omega, J_{22}\right\}$ satisfying
a') $0<T_{22}<1$
b') $J_{22} T_{22} J_{22}=1-T_{22}$
c') $T_{22} u_{22}^{*}\left(1-T_{22}\right)=\left(1-T_{22}\right) u_{22}^{*} T_{22}$

The operator $T$ defined by

$$
\left(\begin{array}{ccc}
0 & 0 & 0 \\
0 & T_{22} & 0 \\
0 & 0 & 1
\end{array}\right)
$$

clearly satisfies a), b) and c).

(ii) $\Rightarrow$ (i). Let $p$ be the projection onto the kernel of $T$. Condition b) implies that the projection $p^{\sharp}=J p J$ is the spectral projection of $T$ corresponding to the eigenvalue 1 . Thus $p$ is neutral. Using projections $p_{1}=p, p_{2}=1-p-p^{\sharp}$ and $p_{3}=p^{\sharp}$, we obtain matrix representaions

$$
T=\left(\begin{array}{ccc}
0 & 0 & 0 \\
0 & T_{22} & 0 \\
0 & 0 & 1
\end{array}\right) \quad \text { and } \quad u=\left(u_{i j}\right)
$$


Then we have

$$
T u^{\sharp}(1-T)=\left(\begin{array}{ccc}
0 & 0 & 0 \\
T_{22} J_{22} u_{32}^{*} J_{31} & T_{22} J_{22} u_{22}^{*} J_{22}\left(1-T_{22}\right) & 0 \\
J_{31} u_{31}^{*} J_{31} & J_{31} u_{21}^{*} J_{22}\left(1-T_{22}\right) & 0
\end{array}\right)
$$

and

$$
(1-T) u^{*} T=\left(\begin{array}{ccc}
0 & u_{21}^{*} T_{22} & u_{31}^{*} \\
0 & \left(1-T_{22}\right) u_{22}^{*} T_{22} & \left(1-T_{22}\right) u_{32}^{*} \\
0 & 0 & 0
\end{array}\right)
$$

According to c) and the fact that $0<T_{22}<1$ on $p_{2} \Re$, we have $u_{21}=0$, $u_{31}=0, u_{32}=0$ and $c^{\prime}$ ). Since $b^{\prime}$ ) follows from $b$ ), $T_{22}$ satisfies $\mathrm{a}^{\prime}$ ), $\mathrm{b}^{\prime}$ ) and $\mathrm{c}^{\prime}$ ) with respect to $u_{22}$. Consequently, $u_{22}$ is quasi-\#-spectral by Lemma 3.3. Thus $u$ is represented by a Tomita's triangular matrix.

Q. E.D.

The following characterization of a bounded \#-unitary $x$ by means of the angular operator $k$ corresponding to the maximal uniformly positive subspace $x \Re^{+}$is more or less known, [1, Theorem 1.5; 4].

Lemma 3.5. For an operator $x$ in $\Omega$ the following two conditions are equivalent:

(i) $x$ is a bounded \#-unitary; and

(ii) there exist an operator $k \in \mathscr{L}\left(\AA^{+}, \AA^{-}\right)$and two unitaries $u^{ \pm} \in \mathscr{L}\left(\Omega^{ \pm}\right)$such that $\|k\|<1$ and

$$
x=S_{k}\left(\begin{array}{cc}
u^{+} & 0 \\
0 & u^{-}
\end{array}\right)
$$

where

$$
S_{k}=\left(\begin{array}{rr}
\left(1-k^{*} k\right)^{-1 / 2} & k^{*}\left(1-k k^{*}\right)^{-1 / 2} \\
k\left(1-k^{*} k\right)^{-1 / 2} & \left(1-k k^{*}\right)^{-1 / 2}
\end{array}\right)
$$

Proof. (i) $\Rightarrow$ (ii). Let $J_{1}=J^{+}, \quad J_{2}=J^{-} \quad$ and $x_{i j}=J_{i} x \mid J_{j}$ \&. The operator $x$ is a bounded \#-unitary if and only if

$$
\begin{aligned}
x_{11}^{*} x_{11}-x_{21}^{*} x_{21} & =1, & x_{11}^{*} x_{12}-x_{21}^{*} x_{22} & =0 \\
\left(-x_{12}^{*} x_{11}+x_{22}^{*} x_{21}\right. & =0) & -x_{12}^{*} x_{12}+x_{22}^{*} x_{22} & =1
\end{aligned}
$$

and 


$$
\begin{aligned}
x_{11} x_{11}^{*}-x_{12} x_{12}^{*}=1, & -x_{11} x_{21}^{*}+x_{12} x_{22}^{*}=0 \\
\left(x_{21} x_{11}^{*}-x_{22} x_{12}^{*}=0\right) & -x_{21} x_{21}^{*}+x_{22} x_{22}^{*}=1 .
\end{aligned}
$$

Among these equalities the first and the third show that $x_{11}$ and $x_{22}$ have bounded inverses. Now we set

$$
k=x_{21} x_{11}^{-1} \text {. }
$$

Since $\quad x_{11}^{*}\left(1-k^{*} k\right) x_{11}=x_{11}^{*} x_{11}-x_{21}^{*} x_{21}=1 \quad$ and $\quad x_{22}^{*}\left(1-k k^{*}\right) x_{22}=x_{22}^{*} x_{22}-$ $\left(x_{22}^{*} x_{21}\right) x_{11}^{-1} x_{11}^{*-1}\left(x_{21}^{*} x_{22}\right)=x_{22}^{*} x_{22}-\left(x_{12}^{*} x_{11}\right) x_{11}^{-1} x_{11}^{*-1}\left(x_{11}^{*} x_{12}\right)=1$ by the second and the third equalities, we find that $1-k^{*} k$ and $1-k k^{*}$ are positive and have bounded inverses. Therefore $\|k\|<1$.

The operators $u^{ \pm}$defined by

$$
u^{+}=\left(1-k^{*} k\right)^{1 / 2} x_{11} \quad \text { and } \quad u^{-}=\left(1-k k^{*}\right)^{1 / 2} x_{22}
$$

satisfy $\left(u^{+}\right)^{*} u^{+}=1$ on $\Omega^{+}$. Thus $u^{+}$is an isometry. Since $1-k^{*} k$ and $x_{11}$ have bounded inverses, $u^{+}$is a unitary on $\Omega^{+}$. Similarly, $u^{-}$ is also a unitary on $\Omega^{-}$. Since $x_{21}=k x_{11}$ and $x_{12}=x_{11}^{*-1}\left(x_{11}^{*} x_{12}\right)$ $=x_{11}^{*-1}\left(x_{21}^{*} x_{22}\right)=k^{*} x_{22}$, we obtain (3.6).

(ii) $\Rightarrow$ (i). By means of $\|k\|<1$ and the identity

$$
k\left(1-k^{*} k\right)^{1 / 2}=\left(1-k k^{*}\right)^{1 / 2} k
$$

we see that $S_{k}$ is a bounded operator and satisfies

$$
\begin{aligned}
S_{k} & =\left(\begin{array}{ll}
1 & k^{*} \\
k & 1
\end{array}\right)\left(\begin{array}{cc}
\left(1-k^{*} k\right)^{-1 / 2} & 0 \\
0 & \left(1-k k^{*}\right)^{-1 / 2}
\end{array}\right) \\
& =\left(\begin{array}{cc}
\left.1-k^{*} k\right)^{-1 / 2} & 0 \\
0 & \left(1-k k^{*}\right)^{-1 / 2}
\end{array}\right)\left(\begin{array}{ll}
1 & k^{*} \\
k & 1
\end{array}\right) .
\end{aligned}
$$

Therefore $S_{k}^{\sharp} S_{k}=S_{k} S_{k}^{\#}=1$ and hence $x^{\sharp} x=x x^{\sharp}=1$.

Q.E. D.

Remark 3.6. (1) In the above lemma the formula (3.6) is nothing but the polar decomposition of $x: x=\left|x^{*}\right| u$. That is $\left|x^{*}\right|=S_{k}$ and

$$
u=\left(\begin{array}{cc}
u^{+} & 0 \\
0 & u^{-}
\end{array}\right) \text {. }
$$

(2) In the above proof (i) $\Rightarrow$ (ii), if we use the fact $[1 ; 6]$ that the operator $S_{k}$ given by the angular operator $k$ corresponding to the maximal uniformly positive subspace $v \Omega^{+}$satisfies $S_{k} J^{ \pm} S_{k}^{-1}$ $=v J^{ \pm} v^{-1}$, then (ii) is immediate.

In the following lemma we shall denote by $\mathscr{L} \mathscr{C}(\mathfrak{S})$ the $C^{*}$-algebra of all compact operators on $\mathfrak{S}$. 
Lemma 3.7. Let $\mathfrak{S}$ be an infinite dimensional Hilbert space and $\mathscr{F}(\mathfrak{S})$ the set of all operators of finite rank. Then the set of $k \in$ $\mathscr{L} \mathscr{C}(\mathfrak{S})$ with $\|k\|<1$ and $\left(1-k^{*} k\right)^{-1 / 2} \in \mathbb{C} 1+\mathscr{F}(\mathfrak{S})$ is norm dense in the set of $k \in \mathscr{L} \mathscr{C}(\mathfrak{S})$ with $\|k\|<1$.

Proof. Consider the correspondence from $\left(1-k^{*} k\right)^{-1 / 2}$ to $k$ for nonnegative $k$ 's. The mapping

$$
h \in\left\{h \in(C 1+\mathscr{L} \mathscr{C}(\mathfrak{S}))_{+}: h \geq 1\right\} \mapsto\left(1-h^{-2}\right)^{1 / 2} \in\left\{k \in \mathscr{L} \mathscr{C}(\mathfrak{E})_{+}:\|k\|<1\right\}
$$

is surjective. Since it is continuous, the image of the subset of $h$ $\in(\boldsymbol{C} 1+\mathscr{F}(\mathfrak{S}))+$ with $h \geq 1$ is dense in the set of $k \in \mathscr{L} \mathscr{C}(\mathfrak{K})+$ with $\|k\|<1$. Namely, the set of $k \in \mathscr{L} \mathscr{C}(\mathfrak{E})+$ with $\left(1-k^{2}\right)^{-1 / 2} \in \mathbb{C} 1+\mathscr{F}(\mathfrak{S})$ is dense in $\left\{k \in \mathscr{L} \mathscr{C}(\mathscr{S})_{+}:|| k i \mid<1\right\}$. For a general $k$ we have only to consider the polar decomposition.

Q. E. D.

Proof of Theorem 3.1. Let $u$ be a bounded \#-unitary with $u$-JuJ compact. Then $u$ is of the form

$$
S_{k}\left(\begin{array}{cc}
u^{+} & 0 \\
0 & u^{-}
\end{array}\right)
$$

and $k \in \mathscr{L}\left(\Omega^{+}, \Omega^{-}\right)$is compact by Lemma 3.5.

Let $\mathscr{L} \mathscr{C}\left(\Omega^{ \pm}\right)$be the $C^{*}$-algebras of compact operators on $\Re^{ \pm}$ and $\mathscr{A}$ the unital $C^{*}$-algebra $\left\{\mathscr{L} \mathscr{C}\left(\Re^{+}\right) \oplus \mathscr{L} \mathscr{C}\left(\Re^{-}\right)\right\}+\mathbb{C} 1_{\Re^{\bullet}}$ Then the group $\mathscr{U}(\mathscr{A})$ of all unitaries in $\mathscr{A}$ is ${ }^{*-s t r o n g l y}$ dense in the group $\mathscr{U}\left(\mathscr{L}\left(\Re^{+}\right) \oplus \mathscr{L}\left(\AA^{-}\right)\right)$by the theorem due to Glimm and Kadison.

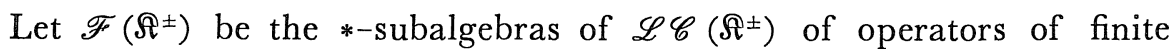
rank and $\mathscr{A}_{0}$ the unital *-subalgebra $\left\{\mathscr{F}\left(\Re^{+}\right) \oplus \mathscr{F}\left(\Re^{-}\right)\right\}+\mathscr{C} 1_{\Re}$. Then $\mathscr{A}_{0}$ is norm dense in $\mathscr{A}$ and the group $\mathscr{U}\left(\mathscr{A}_{0}\right)$ is norm dense in $\mathscr{U}(\mathscr{A})$. Therefore $\mathscr{U}\left(\mathscr{A}_{0}\right)$ is $*$-strongly dense in $\mathscr{U}\left(\mathscr{L}\left(\AA^{+}\right) \oplus \mathscr{L}\left(\Re^{-}\right)\right)$.

Let $\mathscr{L} \mathscr{C}_{1}\left(\AA^{+}, \Re^{-}\right)$be the set of all compact operators $k$ of $\Omega^{+}$to $\AA^{-}$with $\|k\|<1$, and $\mathscr{L} \mathscr{C}_{0}\left(\Re^{+}, \Re^{-}\right)$the set of all $k \in \mathscr{L} \mathscr{C}_{1}\left(\Re^{+}, \Omega^{-}\right)$such that

$$
\left(\begin{array}{cc}
\left(1-k^{*} k\right)^{-1 / 2} & 0 \\
0 & \left(1-k k^{*}\right)^{-1 / 2}
\end{array}\right) \in \mathscr{A}_{0}
$$

Since $k\left(1-k^{*} k\right)^{-1 / 2}=\left(1-k k^{*}\right)^{-1 / 2} k$, the condition that $\left(1-k^{*} k\right)^{-1 / 2}$ belongs to $\mathscr{F}\left(\Re^{+}\right)+C 1$ is equivalent to the condition that $\left(1-k k^{*}\right)^{-1 / 2}$ belongs to $\mathscr{F}\left(\Omega^{-}\right)+\mathbb{C}$. Thus the above condition (3.7) is restated as 


$$
\left(1-k^{*} k\right)^{-1 / 2} \in \mathscr{F}\left(\Re^{+}\right)+C 1 .
$$

Here we use Lemma 3.7, which shows that $\mathscr{L} \mathscr{C}_{0}\left(\AA^{+},{\AA^{-}}^{-}\right)$is norm dense in $\mathscr{L} \mathscr{C}_{1}\left(\Re^{+}, \Re^{-}\right)$. Therefore the element

$$
\left(\left(\begin{array}{cc}
u^{+} & 0 \\
0 & u^{-}
\end{array}\right), k\right)
$$

in the topological product space

$\left\{\mathscr{U}\left(\mathscr{L}\left(\Re^{+}\right) \oplus \mathscr{L}\left(\AA^{-}\right)\right), *\right.$-strong top. $\} \times\left\{\mathscr{L} \mathscr{C}_{1}\left(\AA^{+}, \AA^{-}\right)\right.$, norm top. $\}$ is approximated by a net

$$
\left\{\left(\left(\begin{array}{cc}
u_{i}^{+} & 0 \\
0 & u_{i}^{-}
\end{array}\right), k_{i}\right): i \in I\right\}
$$

in the dense subspace $\mathscr{U}\left(\mathscr{A}_{0}\right) \times \mathscr{L} \mathscr{C}_{0}\left(\Re^{+}, \Re^{-}\right)$. Since $\|k\|<1$, we may assume that inequalities $\left\|k_{i}\right\|<1$ hold for all $i \in I$ by the Kaplansky's density theorem. Here we set

$$
u_{i}=S_{k_{i}}\left(\begin{array}{cc}
u_{i}^{+} & 0 \\
0 & u_{i}^{-}
\end{array}\right) \text {. }
$$

Then $u_{i}$ are bounded \#-unitaries by Lemma 3.5. By virtue of (3.7), the diagonal part of $u_{i}$ belongs to $\mathscr{A}_{0}$ and the off diagonal components are operators of finite rank. Moreover, the diagonal part of $u_{i}$ converges $*$-strongly to that of $u$ and the off diagonal components of $u_{i}$ converge in norm to those of $u$. Thus $u_{i}$ converges *-strongly to $u$.

As $u_{i}$ is of finite rank (mod. $C 1$ ), it is represented by a Tomita's triangular matrix by Lemma 3.2. Therefore there exists for each $u_{i}$ an operator $T_{i}$ satisfying conditions a), b) and c) in Lemma 3.4. By means of condition a) the set $\left\{T_{i}: i \in I\right\}$ is relatively weakly compact, it contains a weakly convergent net $\left\{T_{j}: j \in I^{\prime}\right\}$. Clearly, the weak limit $T$ satisfies conditions a) and b). According to Lemma 3.4 it remains to show that $T$ also satisfies condition c).

Now, rewrite the above weakly convergent net by $\left\{T_{i}: i \in I\right\}$. Then condition c) for $T_{i}$ is equivalent to

$$
u_{i}^{*} T_{i}-T_{i} u_{i}^{\sharp}=T_{i}\left(u_{i}^{*}-u_{i}^{\sharp}\right) T_{i} .
$$

The left hand side is written in the form

$$
\left(u_{i}^{*}-u^{*}\right) T_{i}+\left(u^{*} T_{i}-T_{i} u^{\sharp}\right)+T_{i}\left(u^{\sharp}-u_{i}^{\sharp}\right) .
$$

Since $u_{i}$ converges $*$-strongly to $u$, the first and the last terms 
converge weakly to zero. Therefore the left hand side converges weakly to $u^{*} T-T u^{\sharp}$. Thus our problem is reduced to show that the right hand side converges weakly to $T\left(u^{*}-u^{\sharp}\right) T$. Using $J_{1}=J^{+}$and $J_{2}=J^{-}$, we denote

$$
b_{i j}=J_{i} b \mid J_{j} \Omega \quad \text { for } \quad b \in \mathscr{L}(\Re) .
$$

Then $T\left(u^{*}-u^{*}\right) T=\{T(u-J u J) T\} *$ and

$$
u-J u J=2\left(\begin{array}{cc}
0 & u_{12} \\
u_{21} & 0
\end{array}\right) .
$$

The $u_{i}$ 's have the same type of matrix representation. Thus we can concentrate our attention on the off diagonal elements.

Since $u^{-} J u J$ is compact by assumption, for any $\varepsilon>0$ there exists an operator of finite rank

$$
k=\left(\begin{array}{cc}
0 & k_{12} \\
k_{21} & 0
\end{array}\right)
$$

with $\|(u-J u J)-k\|<\varepsilon / 8$. For the notational convenience we write the off diagonal components by

$$
v_{i}=u_{i}-J u_{i} J, \quad v=u-J u J .
$$

Then

$$
\begin{array}{r}
T_{i} v_{i} T_{i}-T v T=T_{i}\left(v_{i}-v\right) T_{i}+\left(T_{i}-T\right)(v-k) T_{i} \\
+\left(T_{i}-T\right) k T_{i}+T v\left(T_{i}-T\right) .
\end{array}
$$

Here we use the fact that $v_{i}$ converges in norm to $v$, that $T_{i}$ converges weakly to $T$ and that $k$ is of finite rank. Then for $\varepsilon>0$ given in the above and for any unit vectors $\xi, \eta$ in $\Omega$, there exists an $i_{0} \in I$ such that if $i \geq i_{0}$ then

$$
\begin{gathered}
\left\|v_{i}-v\right\|<\varepsilon / 4 \\
\left|\left(\left(T_{i}-T\right) k T_{i} \xi \mid \eta\right)\right|<\varepsilon / 4
\end{gathered}
$$

and

$$
\left|\left(\left(T_{i}-T\right) \xi \mid v^{*} T \eta\right)\right|<\varepsilon / 4 .
$$

Thus, for $i \geq i_{0}$, we have

$$
\left|\left(\left(T_{i} v_{i} T_{i}-T v T\right) \xi \mid \eta\right)\right|<\varepsilon,
$$

which means that $T_{i} v_{i} T_{i}$ converges weakly to $T v T$.

Q.E.D. 


\section{§4. Supplementary Discussions}

1. Let $u$ be a bounded \#-unitary. Suppose that $u$ is quasi-\#spectral with respect to a \#-unitary $v$. Then the closure $w$ of $v u v^{-1} \mid D_{0}$ is unitary, where $D_{0}=v D\left(v^{*} v\right)$. It is then easy to see that $D\left(v^{*} v\right)$ is contained in $D\left(v^{\sharp} w v\right)$ and

$$
v^{\sharp} w v=u \text { on } D\left(v^{*} v\right) \text {. }
$$

Since $u$ is bounded, it coincides with the closure of $v^{\sharp} w v \mid D\left(v^{*} v\right)$. Therefore the \#-unitary $u$ is reconstructed from the unitary $w$, the closure of $v u v^{\sharp} \mid D_{0}$. Thus all the informations of $u$ seem to be contained in w. However there exist examples of bounded \#-unitaries with $\operatorname{Sp}(u) \neq \mathrm{Sp}(w)$ as shown in below, if $v$ is unbounded. This means that the informations of $u$ are contained in a pair of $w$ and $v$, although the quasi-\#-spectrality does not preserve the spectrum. Of course, the \#-spectrality preserves the spectrum.

We begin by discussing the general circumstances. Suppose that a bounded \#-unitary $u$ is quasi-\#-spectral with respect to a \#-unitary v. Namely, the closure of $v u v^{\#} \mid D_{0}$ is unitary as well as \#-unitary. Denote by $\AA_{v}$ the completion of $D\left(v^{*} v\right)$ with respect to the inner product $(\mid)_{0}$ defined by

$$
(\xi \mid \eta)_{v}=(v \xi \mid v \eta) .
$$

Then we have the following commutative diagram:

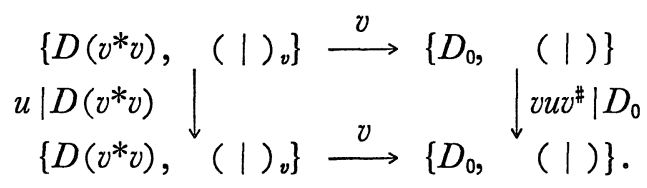

That $u$ is quasi-\#-spectral is then equivalent to that the closure of $u \mid D\left(v^{*} v\right)$ in $\AA_{v}$ is unitary. Moreover the spectrum of the closure of $v u v^{\sharp} \mid D_{0}$ in $\Re$ coincides with that of the closure of $u \mid D\left(v^{*} v\right)$ in $\AA_{v}$. It is worth while noticing that $\Re=\Re_{v}$ whenever $v$ is bounded. If $v$ is unbounded, then $\Re_{v}$ is different from $\Omega$ and it may happen that the spectrum of $u$ in $\AA$ and that of the closure of $u \mid D\left(v^{*} v\right)$ in $\AA_{v}$ are different as shown in the following example.

We begin by giving a definition of a type of operators considered in Example. Let $\left\{\varepsilon_{n}: n \in Z\right.$ Z be the canonical basis of $\ell^{2}(\boldsymbol{Z})$ with 
$\varepsilon_{n}(m)=\delta_{n m}$. Let $x$ and $y$ be the shift and the multiplication operators on $\ell^{2}(\mathbb{Z})$ such that

$$
x \varepsilon_{n}=\varepsilon_{n+1} \quad \text { and } \quad y \varepsilon_{n}=\alpha_{n} \varepsilon_{n},
$$

where $\alpha_{n} \in \mathbb{R}$ with $\alpha_{n}>0$ and $(\|y\|=) \sup _{n \in \boldsymbol{Z}}\left|\alpha_{n}\right|<\infty$. Let $w$ be the closure of $y^{-1} x y$. Then the polar decomposition is of the form

$$
w=x|w| \text { and }|w|=\left(x^{*} y^{-1} x\right) y .
$$

Thus $w$ is a weighted shift:

$$
w \varepsilon_{n}=\left(\alpha_{n} / \alpha_{n+1}\right) \varepsilon_{n+1}, n \in \mathbb{Z},
$$

whose absolute value is interpreted as a kind of difference operator of $y$. It is easy to see that

$$
w^{m} \varepsilon_{n}=\left(\alpha_{n} / \alpha_{n+m}\right) \varepsilon_{n+m}
$$

Example 4.1. We will give a bounded \#-unitary $u$ such that it is quasi-\#-spectral and the spectrum contains the set

$$
\Gamma=\left\{\lambda \in \mathbb{C}:|\lambda|=2 \text { or } 2^{-1}\right\} .
$$

This indicates that the quasi-\#-spectrality does not preserve the spectrum, for the spectrum of a unitary operator must be contained in the unit circle in $\boldsymbol{C}$.

Let $\alpha_{n}=2^{-|n|}$ for all $n \in \mathbb{Z}$ in the above operator. Then $w$ is a weighted shift

$$
w \varepsilon_{n}= \begin{cases}2 \varepsilon_{n+1} & \text { if } n \geq 0 \\ 2^{-1} \varepsilon_{n+1} & \text { if } n \leq-1 .\end{cases}
$$

The spectrum of $w$ contains the set $\Gamma$. Indeed, the spectrum of $w$ is invariant under the multiplication by $\lambda \in \mathbb{C},|\lambda|=1$ and the spectral radiuses of $w$ and $w^{-1}$ are given by

$$
r(w)=\lim _{n \rightarrow \infty}\left\|w^{n}\right\|^{1 / n}=2 \quad \text { and } \quad r\left(w^{-1}\right)=2,
$$

because $\left\|w^{n}\right\|=\left\|w^{-n}\right\|=2^{n}$. Moreover, the spectrum of $w^{*-1}$ also contains $\Gamma$, for $w^{*-1}=x|w|^{-1}$.

Now, let $\Re=\ell^{2}(\boldsymbol{Z}) \oplus \ell^{2}(\boldsymbol{Z})$,

$$
J=\left(\begin{array}{ll}
0 & 1 \\
1 & 0
\end{array}\right), \quad u=\left(\begin{array}{cc}
w & 0 \\
0 & w^{*-1}
\end{array}\right) \text { and } \quad v=\left(\begin{array}{cc}
y & 0 \\
0 & y^{-1}
\end{array}\right) .
$$

Then $\{\Re, J\}$ is a Krein space, in which $u$ and $v$ are \#-unitaries such 
that $u$ is bounded and $D_{0}=v D\left(v^{*} v\right)=R(y) \oplus y^{-1} R\left(y^{2}\right)$. Since each $\varepsilon_{n}$ belongs to $R(y)$ and $y w y^{-1}$ satisfies

$$
y w y^{-1} \varepsilon_{n}=\varepsilon_{n+1}, \quad n \in Z,
$$

the closure of $y w y^{-1} \mid R(y)$ is the shift $x$. Since $y \varepsilon_{n} \in R\left(y^{2}\right), w^{*-1} y \varepsilon_{n}$ $\in R(y)$ and

$$
y^{-1} w^{*-1} y \varepsilon_{n}=\varepsilon_{n+1}, \quad n \in Z,
$$

the closure of $y^{-1} w^{*-1} y \mid y^{-1} R\left(y^{2}\right)$ is also $x$. Therefore the bounded \#-unitary $u$ is quasi-\#-spectral with respect to $v$ and the spectrum of $u$ contains $\Gamma$. Indeed, the closure of

$$
v u v^{\sharp} \mid D_{0}=\left(y w y^{-1} \mid R(y)\right) \oplus\left(y^{-1} w^{*-1} y \mid y^{-1} R\left(y^{2}\right)\right)
$$

is a unitary $x \oplus x$, which has the absolutely continuous spectrum $\{\lambda$ $\in \boldsymbol{C}:|\lambda|=1\}$ with multiplicity 2 .

It should be noted that each point in the unit circle in $\boldsymbol{C}$ belongs to the point spectrum of $w^{*-1}$. Indeed, if we define $\xi_{\lambda}$ by

$$
\xi_{\lambda}=\sum_{n \in Z} \lambda^{n} y \varepsilon_{n}, \quad|\lambda|=1,
$$

then $\xi_{\lambda} \in \ell^{2}(Z)$ and it satisfies

$$
w^{*-1} \xi_{\lambda}=\sum_{n \in Z} \lambda^{n} w^{*-1} y \varepsilon_{n}=\sum_{n \in Z} \lambda^{n} y x \varepsilon_{n}=\bar{\lambda} \xi_{\lambda} .
$$

On the other hand, put

$$
\eta_{n}=n^{-1 / 2}\left(\varepsilon_{1}+\varepsilon_{2}+\cdots+\varepsilon_{n}\right) .
$$

Then $\left\|\eta_{n}\right\|=1$ and $\eta_{n} \in R(y)$. Since

$$
w \eta_{n}-2 \eta_{n}=2 n^{-1 / 2}\left(\varepsilon_{n+1}-\varepsilon_{1}\right),
$$

2 belongs to the approximate point spectrum of $w$. Similarly, we can know that $\Gamma$ is also contained in the approximate point spectrum of $w$.

Remark ([Referee]). By virtue of Theorem 5 in Shield's article [10] it is known that

$$
\operatorname{Sp}(w)=\left\{z \in C: r\left(w^{-1}\right)^{-1} \leq|z| \leq r(w)\right\}
$$

for a (general) invertible bilateral weighted shift $w$.

2. To see the distinction between our result and the theory of Krein-Langer [6], we will consider the finite dimensional case. 
Let $u$ be a \#-unitary. When a Krein space is finite dimensional, there exists an isomorphism $a$ of $\left\{\Re,\langle,\rangle_{\Re}\right\}$ onto $\left\{\mathbb{C}^{N},\langle,\rangle_{\mathbb{C}^{N}}\right\}$ $(N=\operatorname{dim} \Re)$ such that

$$
\begin{aligned}
& a\{u, J\} a^{-1}=\left(\sum_{i=1}^{n} \sum_{j=1}^{n_{j}}\left\{\lambda_{i} S_{i j}, J_{i j}\right\}\right) \\
&\left.\oplus\left(\sum_{i=1}^{m} \oplus \sum_{j=1}^{m_{j}} \oplus\left(\begin{array}{cc}
\mu_{i} S_{n+i, j} & 0_{d_{n+i, j}} \\
0_{d_{n+i, j}} & \overline{\mu_{i}^{-1}} S_{n+i, j}
\end{array}\right), J_{n+i, j}\right\}\right),
\end{aligned}
$$

where $1_{d_{i j}}$ and $0_{d_{i j}}$ are the $d_{i j} \times d_{i j}$ identity and zero matrices $(N=$ $\left.\sum_{i=1}^{n} \sum_{j=1}^{n_{j}} d_{i j}+2 \sum_{i=1}^{m} \sum_{j=1}^{m_{j}} d_{n+i, j}\right)$ and

$$
\begin{aligned}
& \operatorname{Sp}(u) \cap \mathbb{T}=\left\{\lambda_{1}, \lambda_{2}, \ldots, \lambda_{n}\right\}, \\
& \operatorname{Sp}(u) / \mathbb{T}=\left\{\mu_{1}, \mu_{2}, \ldots, \mu_{m}\right\} \cup\left\{\bar{\mu}_{1}^{-1}, \bar{\mu}_{2}^{-1}, \ldots, \bar{\mu}_{m}^{-1}\right\} \text {, } \\
& J_{i j}=\sigma_{i}\left({ }^{\circ} \circ^{1}\right) \text { for } \sigma_{i}=\left\{\begin{array}{l}
1 \text { or }-1 \text { for } i=1, \ldots, n \\
1 \text { for } i=n+1, \ldots, n+m
\end{array}\right.
\end{aligned}
$$

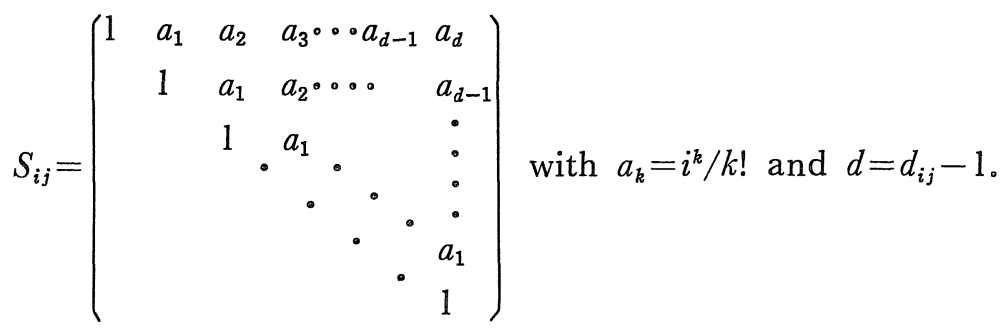

Therefore one of the maximal neutral projections $p$ invariant under $u$ and $u^{\#}$ is of the form

$$
a p a^{-1}=\sum_{i=1}^{n} \sum_{j=1}^{n_{i}} q_{i j}+\sum_{i=1}^{m} \sum_{j=1}^{m_{i}} q_{n+i_{.} j}
$$

where

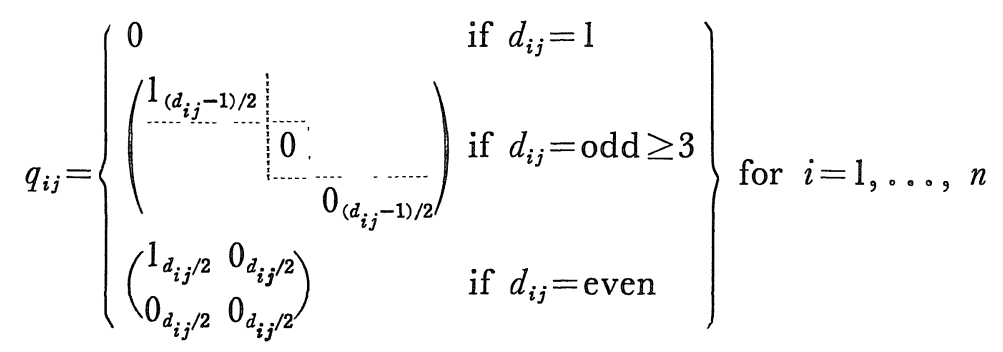

and 


$$
q_{i j}=\left(\begin{array}{ll}
1_{d_{i j}} & 0_{d_{i j}} \\
0_{d_{i j}} & 0_{d_{i j}}
\end{array}\right) \text { for } i=n+1, \ldots, n+m .
$$

Thus we have

$$
a\left(1-p-p^{\#}\right) a^{-1}=\sum_{i=1}^{n} \sum_{j=1}^{n_{i}} r_{i j}
$$

where

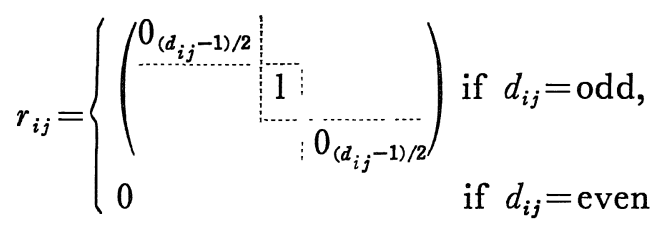

and hence

$$
v \dot{u}_{22} v^{\#}=\sum_{i=1}^{n} \lambda_{i}\left(\sum_{j=1, d_{i j}=\text { odd }}^{n_{i}} p_{i j}\right)
$$

for some \#-unitary $v$ on $\left(1-p-p^{\#}\right) \AA$, where $p_{i j}$ are one dimensional projections.

3. Finally we will consider the problem when the both $(1,1)$ and $(3,3)$ components and the $(2,2)$ component in the Tomita's triangular matrix are orthogonal. We notice that the Gupta-Bleuler triplet due to Araki [3] gives a similar matrix representation of a group if we apply it to our more restrictive situation. This is the case the subspaces corresponding to the $(1,1),(2,2)$ and $(3,3)$ components are interpreted to be the spaces of the longitudinal, transversal and scalar photons, respectively.

Let $u$ and $J$ be a bounded \#-unitary and a metric operator of the forms

$$
\left(\begin{array}{ccc}
u_{11} & u_{12} & u_{13} \\
0 & u_{22} & u_{23} \\
0 & 0 & u_{33}
\end{array}\right) \text { and }\left(\begin{array}{ccc}
0 & 0 & J_{13} \\
0 & J_{22} & 0 \\
J_{31} & 0 & 0
\end{array}\right)
$$

Then $u^{\sharp}$ is of the form

$$
\left(\begin{array}{ccc}
J_{13} u_{33}^{*} J_{31} & J_{13} u_{23}^{*} J_{22} & J_{13} u_{13}^{*} J_{13} \\
0 & J_{22} u_{22}^{*} J_{22} & J_{22} u_{12}^{*} J_{13} \\
0 & 0 & J_{31} u_{11}^{*} J_{13}
\end{array}\right)
$$


Thus $u u^{\sharp}=u^{\sharp} u=1$ is equivalent to the following conditions

$$
\begin{aligned}
& u_{11} J_{13} u_{33}^{*} J_{31}=J_{13} u_{33}^{*} J_{31} u_{11}=1 \\
& u_{22} \text { is a \#-unitary } \\
& u_{12}+u_{11} J_{13} u_{23}^{*} J_{22} u_{22}=0 \\
& u_{11} J_{13} u_{13}^{*}+u_{12} J_{22} u_{12}^{*}+u_{13} J_{31} u_{11}^{*}=0 .
\end{aligned}
$$

Now, let $p$ be a neutral projection and $p_{1}=p, p_{2}=1-p-p^{\#}$ and $p_{3}=p^{\sharp}$. If $x \in \mathscr{L}\left(p_{2} \Re, p_{1} \Re\right)$ and $x_{13} \in \mathscr{L}\left(p_{3} \Re, p_{1} \Re\right)$ with

$$
x_{13}+J_{13} x_{13}^{*} J_{13}=-x J_{22} x^{*} J_{13}
$$

then the matrix

$$
\left(\begin{array}{ccc}
1 & x & x_{13} \\
0 & 1 & -J_{22} x^{*} J_{13} \\
0 & 0 & 1
\end{array}\right)
$$

is a bounded \#-unitary. Of course, we can choose $-(1 / 2) x J_{22} x^{*} J_{13}$ as $x_{13}$.

Proposition 4.2. Let $\left(u_{i j}\right)$ be a Tomita's triangular matrix of a bounded \#-unitary. If there exists an $x \in \mathscr{L}\left(p_{2} \Re, p_{1} \Re\right)$ with $u_{11} x-x u_{22}=$ $u_{12}$, then there exists a bounded \#-unitary $v$ such that

$$
v\left(u_{i j}\right) v^{\#} \simeq\left(\begin{array}{ccc}
u_{11} & 0 & * \\
0 & u_{22} & 0 \\
0 & 0 & u_{33}
\end{array}\right)
$$

Proof. Choose $v$ to be the matrix of the form (4.3) with $x_{13}=$ $-(1 / 2) x J_{22} x^{*} J_{13}$. Q.E. D.

In this case, the $(1,3)$ component in the above matrix is given by

$$
(1 / 2)\left(u_{11} x J_{22} x^{*} J_{13}+2 x u_{22} J_{22} x^{*} J_{13}+x J_{22} x^{*} J_{13} u_{33}\right) \text {. }
$$

If a \#-unitary $u$ is of the form (4.2), then the diagonal part

$$
\left(\begin{array}{ccc}
u_{11} & 0 & 0 \\
0 & u_{22} & 0 \\
0 & 0 & u_{33}
\end{array}\right)
$$

is also \#-unitary and $u$ is expressed as a product of \#-unitaries of the forms (4.4) and (4.3): 


$$
\left(\begin{array}{ccc}
u_{11} & 0 & 0 \\
0 & u_{22} & 0 \\
0 & 0 & u_{33}
\end{array}\right)\left(\begin{array}{ccc}
1 & x & x_{13} \\
0 & 1 & -J_{22} x^{*} J_{13} \\
0 & 0 & 1
\end{array}\right)
$$

where $x=u_{11}^{-1} u_{12}, x_{13}=u_{11}^{-1} u_{13}$.

\section{Acknowledgement}

The author would like to thank Professors $H$. Araki for his valuable discussion on indefinite inner product spaces, A. Ikunishi for his critical reading of the manuscript and the referee for his valuable advice by which Definition 2.3, the proof of Theorem 3.1 and Section 4 are improved.

\section{References}

[1] Ando, T., Linear operators on Krein spaces, Mimeographed note, Hokkaido, Univ., 1979.

[2] Araki, H., On a pathology in indefinite metric inner product space, Commun. Math. Phys., 85 (1982), 121-128.

[3] - Indecomposable representations with invariant inner product - a theory of the Gupta-Bleuler triplet -, Commun. Math. Phys. Vol. 97 (1985), 149-159.

[4] Bognár, J., Indefinite inner product spaces, Springer-Verlag, Berlin and New York, 1974.

[5] Gohberg, I., Lancaster, P. and Rodman, L., Matrices and indefinite scalar product, Birkhäuser Verlag, Basel, Boston and Stuttgart, 1983.

[6] Langer, H., Spectral functions of definitizable operators in Krein spaces, Lecture Notes in Math., 948 (1982), 1-46, Springer Verlag, Berlin, Heiderberg and New York.

[7] Iohvidov, I. S., Krein, M. G. and Langer, H., Introduction to the spectral theory of operators in spaces with an indefinite metric, Akademie-Verlag, Berlin, 1982.

[8] Nakagami, Y., Spectral analysis in Krein spaces, Sixth-seventh seminar on applied functional analysis-information theory and related topics, Ed. by H. Umegaki, (1984), 57-61.

[9] Spectral theory on indefinite inner product spaces, Some topics on functional analysis, Ed. by O. Takenouchi, (1985), 51-82. (in Japanese)

[10] Shields, A. L., Weighted shift operators and analytic function theory, Topics in operator theory, Ed. by C. Pearcy, AMS Mathematical Survay 13 (1974), 49-128b.

[11] Tomita, M., Operators and operator algebras in Krein spaces, I. Spectral analysis in Pontrjagin spaces, RIMS-Kōkyüroku 398, Kyoto Univ. (1980), 131-158. 